\title{
The characteristics of shock wave ignition about nonideal explosives
}

\author{
Quanhu Lei ${ }^{1}$, Penggang Jin², Jian Yang ${ }^{3}$, Songtao Ren ${ }^{4}$, Hongtao Xu${ }^{5}$, Hongbin $\mathrm{Li}^{6}$, \\ Xibo Jiang ${ }^{7}$ \\ Xi'an Modern Chemistry Research Institute, Xi'an, China \\ ${ }^{2}$ Corresponding author \\ E-mail: 1'leiquanhu204@163.com,2jinpenggang204@126.com,3yangj_2@163.com, \\ rengshongtao@163.com, ${ }^{5} x u h o n g t a o @ 163 . c o m,{ }^{6}$ lihongbing@163.com,7jiangxibo@163.com
}

Received 14 January 2021; received in revised form 20 January 2021; accepted 27 January 2021 DOI https://doi.org/10.21595/vp.2021.21873

Check for updates

Copyright $(2021$ Quanhu Lei, et al. This is an open access article distributed under the Creative Commons Attribution License, which permits unrestricted use, distribution, and reproduction in any medium, provided the original work is properly cited.

\begin{abstract}
The ignition and detonation process of non-ideal explosives is orders of magnitude different from that of ideal explosives on the time scale, which makes non-ideal explosives have a longer growth distance when subjected to shock stimulation, and the ignition growth process of non-ideal explosives is strongly dependent on external conditions, and the process involves complex physical and chemical changes of explosive materials, and today's technology cannot accurately characterize it. In order to study the ignition growth characteristics of non-ideal explosives below the critical initiation pressure, by measuring the pressure change process in the explosive inside the sample tube during the deformation stage of the shock wave sensitivity, it is found that the internal pressure of the explosive first increases and then decays. Therefore, it is judged that when the critical initiation pressure of the explosive is lower than the critical ignition pressure, the non-ideal explosive still has the next critical ignition pressure. This lower pressure can cause the ignition reaction in the non-ideal explosive to occur locally and form a pressure increase phenomenon in the explosive.
\end{abstract}

Keywords: shock wave sensitivity, shock wave ignition, explosive safety.

\section{Introduction}

Explosive impact initiation characteristics are usually characterized by gap experiments in the industry [1-3]. With the development of high-pressure testing technology [4, 5], a method of using critical initiation pressure to indicate the safety of explosives under impact has gradually emerged [6]. This method obtains the critical pressure of a large number of explosive charges. At this stage, when the safety of explosives under impact is involved, the critical initiation pressure is used as the ignition initiation condition in most numerical simulations at home and abroad [7-10].

However, in the gap test of non-ideal explosives [11, 12], a phenomenon clearly different from that of ideal explosives can be observed. In the non-ideal explosive Langley method experiment, it was found that some samples were judged to be undetonated, and the steel tube filled with the sample was significantly deformed from the detonation end, but this deformation started from the detonation end and ended at the sample in the middle and lower section of the tube, the sample tube at the bottom is not deformed. This deformation process causes internal damage to the explosive. There are some reports on the impact of the damage on the explosive initiation characteristics, but there are no reports about this unconventional phenomenon.

In this paper, by measuring the pressure change characteristics of the explosive charge in the deformation stage of the sample tube, it is concluded that the explosive charge has been ignited under this pressure. At the same time, it is proposed that the safety judgment of the explosive charge under the impact action should be based on the lower impact critical ignition pressure as the judgment basis. The specialists of National Institute of Technology of India once evaluate the performance of LoRa LPWAN technology for IoT-base blast-induced ground vibration system [13]. 


\section{Experimental design and equipment}

\subsection{Experimental design}

In order to study the mechanism by which the non-ideal explosive is judged to be undetonated but the sample tube is severely deformed in the gap test, a typical non-ideal explosive is selected. First, the gap test is carried out to obtain the critical impact detonation gap thickness. Then, when the thickness of the gap is greater than the thickness of the critical initiation gap, the sample tube is deformed and the pressure growth process in the tested explosive is witnessed under the condition that the plate is not perforated, and the pressure change characteristics at this time are obtained. Foreign experts have used the Internet of Things technology to remotely monitor the seismic waves generated by mine blasting and understand the level of BIGV intensity [13].

\subsection{Experimental samples and their assembly}

A typical non-ideal explosive was selected in the test, and its component was HMX/AL/AP/Binder: 50/26/12/12. The code of the sample was P. The explosive sample was prepared by pouring process and poured directly in a sample tube with a diameter of $25 \mathrm{~mm}$ and a height of $75 \mathrm{~mm}, 10$ samples were prepared for each sample. In the pressure measurement experiment, the manganese-copper sensor was evenly distributed in the entire sample area where the bell mouth shape appeared in the experiment. The details are shown in the following Fig. 1.
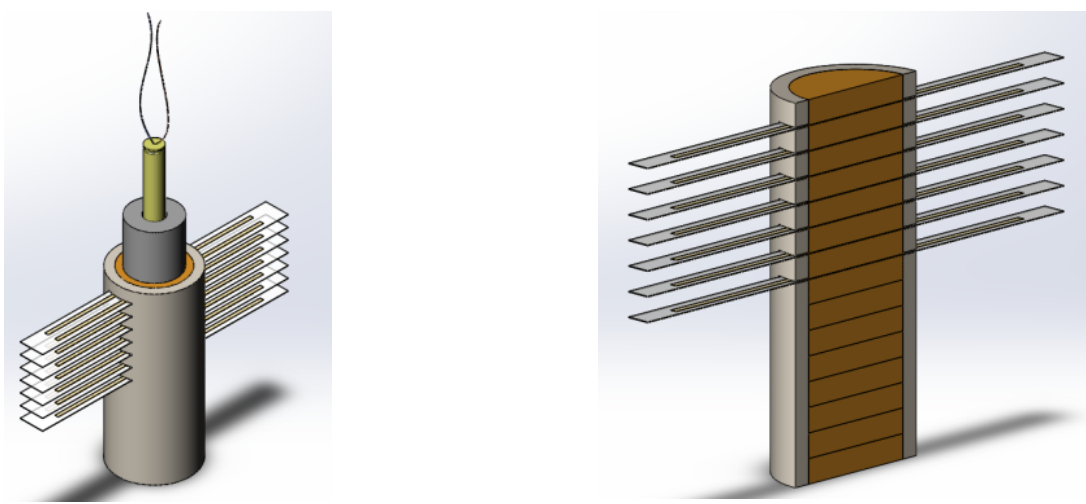

Fig. 1. Sample assembly drawing in pressure measurement

\subsection{Test equipment}

In the gap test, the main charge column is pressed passivation RDX with a density of $(1.60 \pm 0.05) \mathrm{g} / \mathrm{cm}^{3}$ and a diameter of $40 \mathrm{~mm}$; the gap is made of PMMA with a diameter of $(40 \pm 0.1) \mathrm{mm}$.

The pressure measurement device in the charge column consists of a high-speed digital oscilloscope, a dynamic high-pressure sensor, etc. The data recorder is a Tektronix 7504 high-speed digital oscilloscope, the sampling speed is $1 \mathrm{GHz}$, each oscilloscope has four channels, and each test requires two oscilloscopes to collect data at the same time. The dynamic high-pressure sensor adopts the PVDF pressure sensor, manufactured by Dynasen, USA, with a measuring range of $0 \sim 10 \mathrm{GPa}$.

\section{Test results and discussion}

\subsection{Test phenomena and test results}

The assembly of the gap test is shown in Fig. 2. The criterion to judge whether the sample has 
detonation is whether the witness plate at the bottom of the sample is perforated. The typical test photo after detonation is shown in Fig. 3. The condition of the sample tube is not used as the criterion for the results. In the test of non-ideal explosive $\mathrm{P}$, in the experiment without perforation, the sample tube was deformed to varying degrees. As the thickness of the gap of $p$ decreases (increased impact pressure), the deformation of the sample tube increases. As shown in Fig. 4, the deformation of the sample tube in the $42 \mathrm{~mm}$ gap test is almost not observed. When the gap is $40 \mathrm{~mm}$ thick, the sample tube starts Deformation has occurred. Table 1 is a record table of the test process of the non-ideal explosive partition code-named P. It can be seen from the table that the critical gap thickness of non-ideal explosive $\mathrm{P}$ is $26 \mathrm{~mm}$.

Table 1. Gap test records

\begin{tabular}{|c|c|c|c|}
\hline Sample & \multicolumn{3}{|c|}{ P } \\
\hline \multirow{5}{*}{ Experimental records } & Serial number & The thickness of the gap/mm & experimental records \\
\cline { 2 - 4 } & 1 & 40 & $\times$ \\
\cline { 2 - 4 } & 2 & 20 & $\sqrt{ }$ \\
\cline { 2 - 4 } & 3 & 30 & $\times$ \\
\cline { 2 - 4 } & 4 & 25 & $\sqrt{ }$ \\
\cline { 2 - 4 } & 5 & 27 & $\times$ \\
\cline { 2 - 4 } & 6 & 25 & $\times$ \\
\hline Results & 7 & 27 & \multicolumn{3}{|l}{} \\
\hline Note: $\sqrt{\mid}$ : Detonation, $\times$ : Undetonated & \multicolumn{3}{|l}{} \\
\hline
\end{tabular}

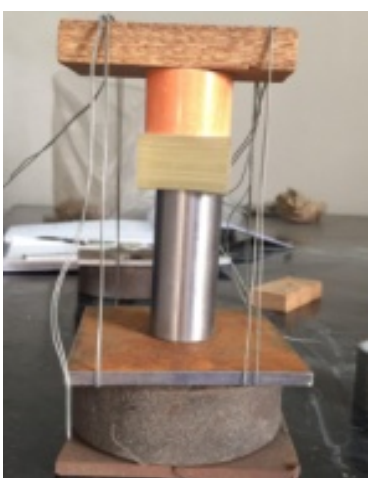

Fig. 2. Gap test assembly drawing



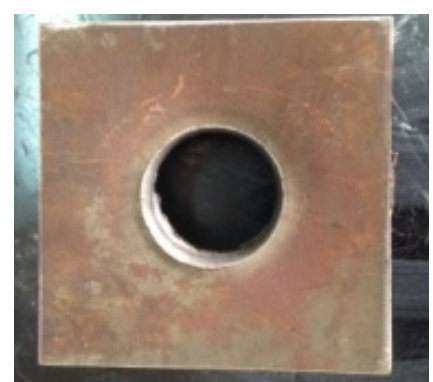

Fig. 3. The witness plate after detonation reaction

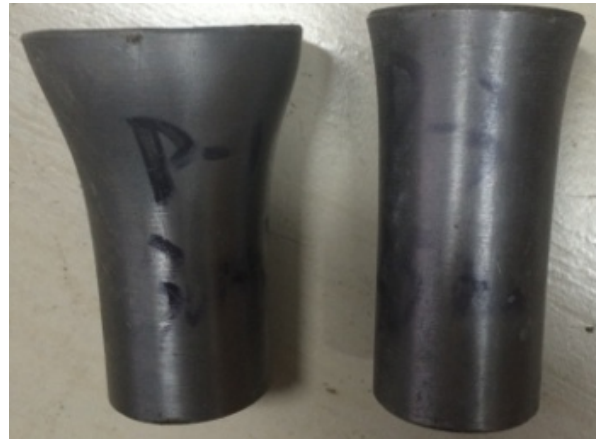

Fig. 4. Nonideal explosive sample tuble picture after Gap test

\subsection{Test results of internal pressure measurement}

It can be seen from the above test that when the thickness of the gap is $40 \mathrm{~mm}$, the sample tube 
begins to deform. Therefore, the sample under the thickness of the gap of $40 \mathrm{~mm}$ is selected to measure the internal pressure of the tested explosive. The pressure curve is shown in Fig. 5.

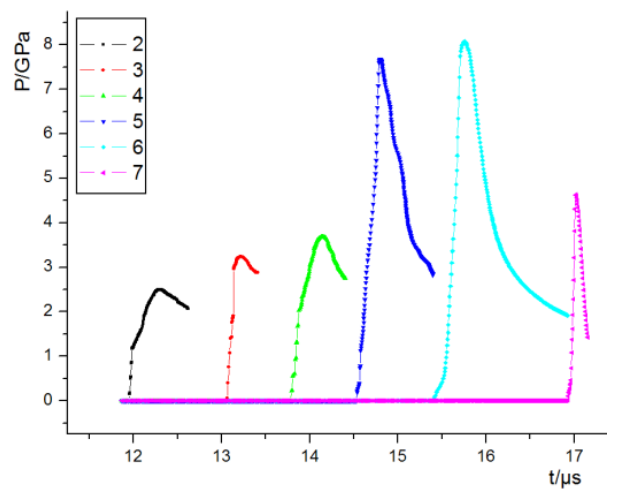

Fig. 5. The pressure curve in non-reactive explosive

It can be seen from Fig. 5 that when the thickness of the gap is $40 \mathrm{~mm}$, the internal pressure of the tested explosive first decreases, then increases and then decreases again. When the thickness of the gap is $50 \mathrm{~mm}$, the sample tube is not deformed, and the pressure in the sample is also measured, the pressure curve is shown in Fig. 6.



Fig. 6. The pressure curve in non-reactive explosive

\subsection{Discussion of results}

It can be seen from Fig. 6 that when the sample tube is not deformed, the shock wave pressure of the sample in the sample tube is gradually decreasing. When the sample tube is deformed, the shock wave pressure in the sample first increases and then decreases again, and finally fails to form a stable pressure increase. According to the law of shock wave propagation in solid media, when the sample does not react, the pressure in the sample should show a gradual decrease trend, while the pressure of the sample in the article has a significant increase process, which shows that part of the sample in the process and make the pressure shows increase. A chemical reaction of the part of sample occurs in the local sample, but a stable detonation process is not formed. At this time, the reaction of the sample can only be called "ignition", but it does not form detonation. For energetic materials, the "ignition" is already unsafe. According to the method 605.1 shock wave sensitivity -gap method of GJB772A, the method cannot determine whether the sample is "ignited", but can only determine the conditions for stable detonation of the sample.

It can be seen from the above analysis that the non-ideal explosive $\mathrm{P}$ has a critical ignition pressure and a critical initiation pressure under the effect of shock waves, and the critical ignition 
pressure is lower than the critical initiation pressure. When the "ignition" phenomenon occurs in energetic materials, the probability of developing into a stable detonation increases significantly and it is already in a very dangerous state. From a safety point of view, the critical "ignition" pressure of the explosive should be obtained. Therefore, the explosive effect on the shock wave, the safety evaluation should use the critical "ignition" pressure instead of the critical detonation pressure. When considering how to ensure the stable detonation of explosives, the critical detonation pressure should be considered as the standard.

Non-ideal explosives have low reaction rate of the AP and AL and other materials in the structure. When the main components such as HMX in non-ideal explosives completely react, these low-reaction rate materials will not completely react be within the reaction time of HMX and other high-speed reaction materials. The inability to complete the reaction results in an increase in the overall reaction time of non-ideal explosives, which is the essential reason for the significant increase in the detonation reaction distance of non-ideal explosives. The detonation growth distance of the non-ideal explosive used in this article is about $13 \mathrm{~mm}$ when the gap thickness is $40 \mathrm{~mm}$, while the detonation growth distance of the ideal explosive is generally on the order of micrometers, which is about thousands of times different. This makes non-ideal explosives have an obvious "ignition" growth process, and ideal explosives have a very short growth distance, and the difference between the critical "ignition" pressure and the critical initiation pressure is not significant, so the 605.1 shock wave sensitivity-gap method of GJB772A does not distinguish.

\section{Conclusions}

Under the effect of shock wave, the critical ignition pressure should be used as the index parameter when assessing the safety of non-ideal explosives, and the critical initiation pressure should be used as the index parameter when assessing the stable detonation of non-ideal explosives.

It can be seen from the experiment that the method 605.1 shock wave sensitivity of GJB772A for non-ideal explosives determines the critical initiation pressure, and the critical ignition pressure cannot be obtained.

Non-ideal explosives have a long detonation growth distance under the effect of shock waves, and their detonation growth distance is thousands of times that of ideal explosives, which results in a significant difference between the critical ignition pressure and critical initiation pressure of non-ideal explosives.

\section{References}

[1] Ma Xiugui The shock wave of explosive -gap test. Chinese Journal of Explosive and Propellants, Vol. 4, 1982, p. 1-6.

[2] Tian Wenhui, He Qizhong, Peng Jinhua Determination of micro-nano RDX shock sensitivity by underwater method. Chinese Journal of Explosive and Propellants, Vol. 36, Issue 4, 2013, p. 29-32.

[3] Lv Chunling, Zhang Jinglin, Wang Jingyu, et al. Selectivity of sub-micron explosive sensitivity to shock wave. Energetic Materials, Vol. 13, Issue 5, 2005, p. 319-321.

[4] Wang Hao, Wang Qinhui, Huang Wenbin, et al. Shock sensitivity of DNTF reduced by using DNAN. Chinese Journal of Energetic Materials, Vol. 18, Issue 4, 2010, p. 435-438.

[5] Peng Qixian, Tan Hua, Wang Wei Surface velocity measurement using all-fiber visar. Chinese Journal of High-Pressure Physics, Vol. 15, Issue 2, 2001, p. 141-145.

[6] Yu Shangjiang, Yang Jixiang, Chen Xian A review on measurement techniques of high dynamic pressure. Protective Engineering, Vol. 33, Issue 4, 2011, p. 65-72.

[7] Huang Yun, Wang $\mathbf{X u}, \mathbf{X u}$ Seng Study on critical initiation pressure of HMX-base propellant. Explosive Materials, Vol. 49, Issue 1, 2020, p. 34-39.

[8] Bai Zhi-ling, Duan Zhuo-ping, Wen Li-jing, et al. A modified of mesoscopic reaction rate model for shock initiation of PBXs. Chinese Journal of Energetic Materials, Vol. 27, Issue 8, 2019, p. 629-635. 
[9] Miao Feichao, Zhou Lin, Zhang Xiangrong, et al. Implantation and application of ignition and growth reaction rate equation in LS-DYNA Software. Acat Armamentarii, Vol. 40, Issue 7, 2019, p. 1411-1417.

[10] Qian Shichuan, Gan Qiang, Ren Zhiwei, et al. Study on one-dimensional shock initiation criterion of HNS-IV explosive. Chinese Journal of Energetic Materials, Vol. 26, Issue 6, 2018, p. 495-501.

[11] Jin Penggang, Jiang Xibo, Li Hongbin, et al. Effects of shock insults on explosive charges shock wave sensitivity. Equipment Environmental Engineering, Vol. 16, Issue 9, 2019, p. 1-4.

[12] Jiang Xibo, Jin Penggang, Wang Jianlin, et al. Damage characteristics of two HMX-based anti-overloaded explosives under shock loading. Chinese Journal of Energetic Materials, Vol. 25, Issue 12, 2017, p. 991-996.

[13] Prashanth Ragam, Nimaje D. S. Performance evaluation of LoRa LPWAN technology for IoT-based blast-induced ground vibration system. Journal of Measurements in Engineering, Vol. 7, Issue 3, 2019 , p. 119-133. 